\title{
Research on Two Kinds of Adaptive Synchronization Method for Uncertain Fourth Order Chaotic Systems based on Sigmoid Function and Soft Function Method
}

\author{
Zhanlei Shang $^{1, \mathrm{a}}$, Jing Cheng ${ }^{2, \mathrm{~b}}$, Junwei Lei ${ }^{3, \mathrm{c}}$ and Ruiqi Wang ${ }^{4, \mathrm{~d}}$ \\ ${ }^{1}$ College of Computer and Communication Engineering, Zhengzhou University of \\ Light Industry, Zhengzhou 450002, China \\ ${ }^{2}$ College of Computer and Communication Engineering, Zhengzhou University of \\ Light Industry, Zhengzhou 450002, China \\ ${ }^{3}$ Department of Control Engineering, Naval Aeronautical and Astronautical \\ University, Yantai Shandong 264001, China \\ ${ }^{4}$ Department of Armament Engineering, Naval Aeronautical and Astronautical \\ University, Yantai Shandong 264001, China \\ shangzl@zzuli.edu.cn, ${ }^{a}$ cjcheng@zzuli.edu.cn, \\ cleijunwei@126.com, ${ }^{d}$ richkey1980@gmail.com,
}

\begin{abstract}
Synchronization of chaotic systems can be used in the secure communication so it greatly attracted interests of many researchers. The bounded characteristics of chaotic system was used and an uniformed adaptive law was designed to solve system uncertainties which make the whole design very simple. And two kinds of functions was used to take place of traditional sign function, then the chattering problem was greatly improved. A Lyapunov function was chosen to guarantee the stability of the whole design. At last, detailed simulation was done to show the rightness and effectiveness of the proposed method.
\end{abstract}

Keywords: Chaotic systems; nonlinear; robust control; adaptive synchronization; uncertainty; desired trajectory

\section{Introduction}

Chaotic system has many advantages such as it has random characteristics and rich dynamic response [1-9], especially it can be used in secure communication with synchronization of two chaotic systems. So it aroused interests of researchers in many countries because of its military application [10-13].

But the system uncertainties will always exist in every real engineering systems, so how to solve uncertainties is the biggest problem that affect synchronization effect [14-17]. And many researchers designed sliding mode controller since it can provide a strong robustness for controllers. But the sliding mode strategy will has a disadvantage that it will cause chattering problems because of the use of sign function. So in this paper, we introduced two kinds of functions to take place of the sign function, one is the soft function and another is the sigmoid function. Simulation result showed that it can improve the synchronization effect and reduce chattering problem obviously.

Also many researchers designed many kinds of adaptive controllers [18-21] by using adaptive strategy and robust design method. But most of the design were based on different structure and different function of systems, so adaptive law was very complex in some situation that subsystems are different from each other. In this paper, a novel design was proposed by using the bounded characteristics of chaotic system that a uniformed formation of adaptive law design method was applied in every subsystem. So the whole 
controller design of very simple although the control object is a fourth order system. So the main advantage of the proposed method is that it is very simple and easy to be applied in control engineering, also it can reduce chattering problem by introducing soft function and sigmoid function.

\section{Problem Description}

Considering the following driver system and response system, where parameters of response system is known and there exists unknown parameters and uncertain nonlinear function in driven system.

The driven system can be written as

$$
\dot{x}=f_{x}(x)+F_{x}(x) \theta_{x}+\Delta(x, t)
$$

The response system can be written as

$$
\dot{y}=f_{y}(y)+b u
$$

Take a four dimension system as a example, the main driven system can be written as

$$
\begin{aligned}
& \dot{x}_{1}=f_{x 1}\left(x_{1}, \cdots, x_{4}\right)+\sum_{j=1}^{p_{1}} F_{x 1 j}\left(x_{1}, \cdots, x_{4}\right) \theta_{x 1 j}+\sum_{j=1}^{q_{1}} \Delta_{x 1 j}(x, t) \\
& \dot{x}_{2}=f_{x 2}\left(x_{1}, \cdots, x_{4}\right)+\sum_{j=1}^{p_{2}} F_{x 2 j}\left(x_{1}, \cdots, x_{4}\right) \theta_{x 2 j}+\sum_{j=1}^{q_{2}} \Delta_{x 2 j}(x, t) \\
& \dot{x}_{3}=f_{x 3}\left(x_{1}, \cdots, x_{4}\right)+\sum_{j=1}^{p_{3}} F_{x 3 j}\left(x_{1}, \cdots, x_{4}\right) \theta_{x 3 j}+\sum_{j=1}^{q_{3}} \Delta_{x 3 j}(x, t) \\
& \dot{x}_{4}=f_{x 4}\left(x_{1}, \cdots, x_{4}\right)+\sum_{j=1}^{p_{3}} F_{x 4 j}\left(x_{1}, \cdots, x_{4}\right) \theta_{x 4 j}+\sum_{j=1}^{q_{3}} \Delta_{x 4 j}(x, t)
\end{aligned}
$$

And the slave response system can be written as

$$
\begin{aligned}
& \dot{y}_{1}=f_{y 1}\left(y_{1}, \cdots, y_{4}\right)+b_{1} u_{1} \\
& \dot{y}_{2}=f_{y 2}\left(y_{1}, \cdots, y_{4}\right)+b_{2} u_{2} \\
& \dot{y}_{3}=f_{y 3}\left(y_{1}, \cdots, y_{4}\right)+b_{3} u_{3} \\
& \dot{y}_{4}=f_{y 4}\left(y_{1}, \cdots, y_{4}\right)+b_{4} u_{4}
\end{aligned}
$$

When $\theta_{x}$ is unknown parameter, and the number of unknown parameter is $\sum_{i=1}^{n} p_{i}$, and the number of uncertain nonlinear function is $\sum_{i=1}^{n} q_{i}, \quad b_{i}$ is a known constant [8-10].

So the robust adaptive control target for chaotic system with unknown parameter and uncertain nonlinear function is to design the control $u_{i}=u_{i}\left(x, y, \hat{r}_{i j}\right), \dot{\hat{r}}_{i j}=f_{i j}\left(z_{1}, z_{2}, z_{3}\right)$ such that the state of slave system can trace state of master system, such as $y \rightarrow x$.

Considering the above adaptive PID control is too complex, so the design of robust adaptive synchronization need benefit the boundedness of chaotic system. And use a bounded function to describe the uncertain region of the unknown information of driven system and response system, then design a robust adaptive controller to synchronize two systems [11-14]. 


\section{Assumption}

Two assumptions are built for the above system to simplify the analysis.

Assumption 1: the driven system and response system have the same structure, it means that it has the same dimension.

Assumption 2: the nonlinear function satisfies the below conditions, for $1 \leq i \leq n$, $1 \leq j \leq p_{2}$, there exists a unknown positive constant $r_{i j} \leq d_{i j}$ such that

$$
\begin{aligned}
& f_{y i}\left(y_{1}, \cdots, y_{4}\right)-f_{x i}\left(x_{1}, \cdots, x_{4}\right)-\sum_{j=1}^{p_{1}} F_{x i j}\left(x_{1}, \cdots, x_{4}\right) \theta_{x i j}-\sum_{j=1}^{q_{1}} \Delta_{x i j}(x, t) \\
\leq & r_{i 1}\left|z_{1}\right|+r_{i 2}\left|z_{2}\right|+r_{i 3}\left|z_{3}\right|
\end{aligned}
$$

where $d_{i j}$ is a known constant. Because the chaotic system is bounded, so it is easy to be satisfied for many chaotic systems [15-17].

\section{Robust Adaptive Synchronization Law Design based on Soft Function Method}

Define the error variable as $z_{i}=y_{i}-x_{i}$, where the error system can be written as

$$
\begin{aligned}
\dot{z}_{i}= & f_{y i}\left(y_{1}, \cdots, y_{4}\right)-f_{x i}\left(x_{1}, \cdots, x_{4}\right) \\
& -\sum_{j=1}^{p_{1}} F_{x i j}\left(x_{1}, \cdots, x_{4}\right) \theta_{x i j}-\sum_{j=1}^{p_{2}} \Delta_{x i j}(x, t)+b_{i} u_{i}
\end{aligned}
$$

Then it also has

$$
z_{i} \dot{z}_{i} \leq r_{i 1}\left|z_{1}\right|\left|z_{i}\right|+r_{i 2}\left|z_{2}\right|\left|z_{i}\right|+r_{i 3}\left|z_{3}\right|\left|z_{i}\right|+z_{i} b_{i} u_{i}
$$

Use the adaptive method to design the $\operatorname{control} u_{i}$ as

$$
u_{i}=b_{i}^{-1}\left[\hat{r}_{i 1}\left|z_{1}\right|+\hat{r}_{i 2}\left|z_{2}\right|+\hat{r}_{i 3}\left|z_{3}\right|\right] \frac{z_{i}}{\left|z_{i}\right|+\varepsilon_{i}}
$$

Where $\frac{z_{i}}{\left|z_{i}\right|+\varepsilon_{i}}$ is a soft function and has a characteristic similar to sign function.

The error of assumption is defined as

$$
\tilde{r}_{i j}=r_{i j}-\hat{r}_{i j}
$$

Solve its derivative as

$$
\dot{\tilde{r}}_{i j}=-\dot{\hat{r}}_{i j}
$$

Considering that if $\varepsilon_{i}$ is a small positive constant, then

$$
\frac{z_{i}}{\left|z_{i}\right|+\varepsilon_{i}} \approx \operatorname{sign}\left(z_{i}\right)
$$

Where the parameter adaptive law is designed as

$$
\dot{\hat{r}}_{i j}=-\left|z_{i} z_{j}\right|
$$


Choose the Lyapunov function as

$$
V_{1}=\sum_{i=1}^{n} z_{i}^{2}+\sum_{j=1}^{j=n} \sum_{i=1}^{i=n} \tilde{r}_{i j}
$$

It is easy to get

$$
\dot{V_{1}} \leq 0
$$

So the system is stable and synchronization is realized [18-21].

\section{Robust Adaptive Synchronization Law Design based on Sigmoid Function Method}

Define the error variable as $z_{i}=y_{i}-x_{i}$, where the error system can be written as

$\dot{z}_{i}=f_{y i}\left(y_{1}, \cdots, y_{4}\right)-f_{x i}\left(x_{1}, \cdots, x_{4}\right)$

$$
-\sum_{j=1}^{p_{1}} F_{x i j}\left(x_{1}, \cdots, x_{4}\right) \theta_{x i j}-\sum_{j=1}^{p_{2}} \Delta_{x i j}(x, t)+b_{i} u_{i}
$$

Then it also has

$$
z_{i} \dot{z}_{i} \leq r_{i 1}\left|z_{1}\right|\left|z_{i}\right|+r_{i 2}\left|z_{2}\right|\left|z_{i}\right|+r_{i 3}\left|z_{3}\right|\left|z_{i}\right|+z_{i} b_{i} u_{i}
$$

Use the adaptive method to design the $\operatorname{control} u_{i}$ as

$$
u_{i}=b_{i}^{-1}\left[\hat{r}_{i 1}\left|z_{1}\right|+\hat{r}_{i 2}\left|z_{2}\right|+\hat{r}_{i 3}\left|z_{3}\right|\right] h_{i}\left(z_{i}\right)
$$

Where $h_{i}\left(z_{i}\right)$ is a sigmoid function and it has a characteristic similar to sign function and it can be written as

$$
h_{i}\left(z_{i}\right)=\frac{1-e^{-\tau_{i} z_{i}}}{1+e^{-\tau_{i} z_{i}}}
$$

The error of assumption is defined as

$$
\tilde{r}_{i j}=r_{i j}-\hat{r}_{i j}
$$

Solve its derivative as

$$
\dot{\tilde{r}}_{i j}=-\dot{\hat{r}}_{i j}
$$

Considering that if $\tau_{i}$ is a big positive constant, then

$$
\frac{1-e^{-\tau_{i} z_{i}}}{1+e^{-\tau_{i} z_{i}}} \approx \operatorname{sign}\left(z_{i}\right)
$$

We can also design adaptive law as

$$
\dot{\hat{r}}_{i j}=-\left|z_{i} z_{j}\right|
$$

Choose the Lyapunov function as

$$
V_{1}=\sum_{i=1}^{n} z_{i}^{2}+\sum_{j=1}^{j=n} \sum_{i=1}^{i=n} \tilde{r}_{i j}
$$


It is easy to get

$$
\dot{V_{1}} \leq 0
$$

So the system is stable and the synchronization is realized according to Lyapunov stability theorem.

\section{Numerical Simulation for Synchronization with Soft Function Method}

We choose a four dimension chaotic system as an example to do the numerical simulation to test the rightness of the proposed method and its mode can be written as

$$
\begin{gathered}
\dot{x}_{1}=a\left(x_{2}-x_{1}\right)+k_{1 b} \sin x_{4} \\
\dot{x}_{2}=b x_{1}-x_{2}-x_{1} x_{3}+k_{2 b} \cos x_{4} \\
\dot{x}_{3}=x_{1} x_{2}-c x_{3}+k_{3 b} \sin \left(x_{4} x_{3}\right) \\
\dot{x}_{4}=\sin \left(x_{1} x_{2}\right)+k_{4 b} \cos \left(x_{3}\right)
\end{gathered}
$$

Where $a=10, b=28, c=\frac{8}{3}, k_{1 b}=0.1, \quad k_{2 b}=0.1, \quad k_{3 b}=0.2, k_{4 b}=-1$, the above system is a chaotic system and it has an attractor. We set initial value as $x_{1}=-0.2, x_{2}=0.7, x_{3}=5, x_{4}=0.2$, then its free movement can be shown as following Figure 1 to Figure 4.

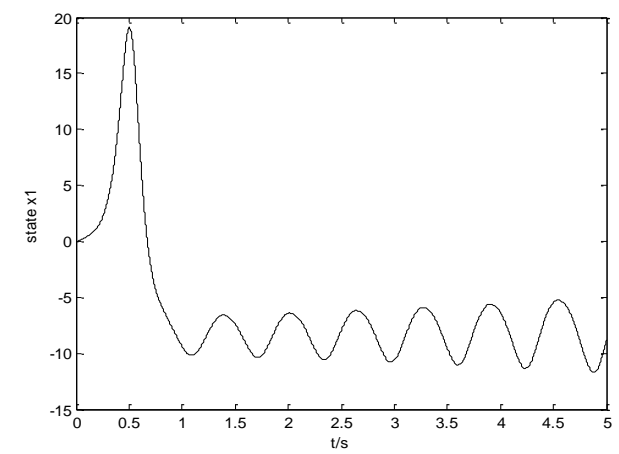

Figure 1. The Curve of State $x 1$

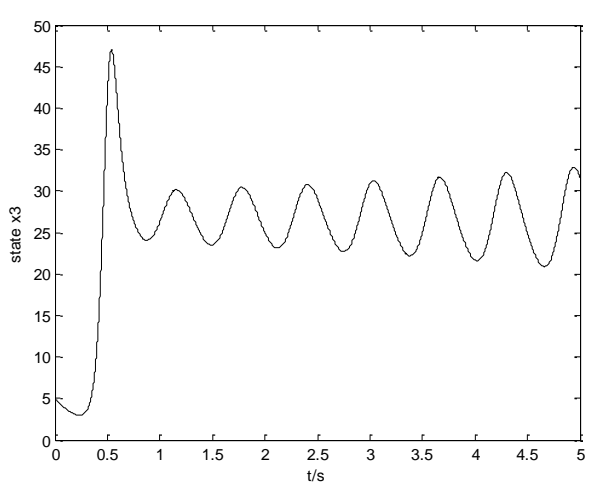

Figure 3. The Curve of State $\times 3$

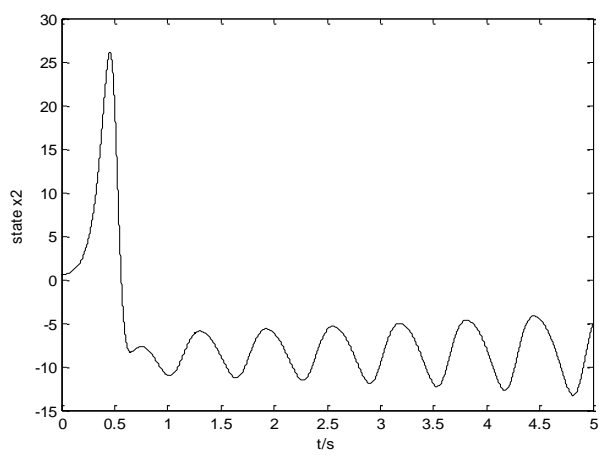

Figure 2. The Curve of State $x 2$

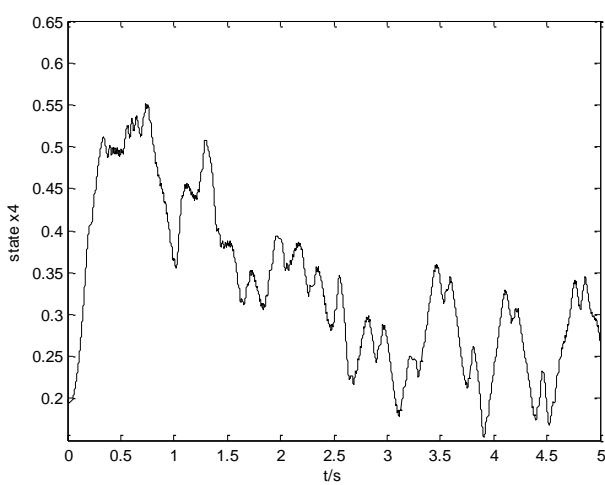

Figure 4. The Curve of State $\times 4$ 
We set $a, b, c$ as unknown parameters and set $k_{l b}$ as coefficient of nonlinear function. The structure of response system is known and it can be described as follows:

$$
\begin{gathered}
\dot{y}_{1}=a_{y}\left(y_{2}-y_{1}\right)+u_{1} \\
\dot{y}_{2}=b_{y} y_{1}-y_{2}-y_{1} y_{3}+u_{2} \\
\dot{y}_{3}=y_{1} y_{2}-c_{y} y_{3}+u_{3} \\
\dot{y}_{4}=\sin \left(y_{1} y_{2}\right)+u_{4}
\end{gathered}
$$

Choose parameter as $a_{y}=9, b_{y}=25, c_{y}=2$, and the initial state of response system is $y_{1}=-0.3, y_{2}=0.5, y_{3}=3, y_{4}=0.7$, use above robust adaptive strategy with soft function method, the simulation result is as follows:

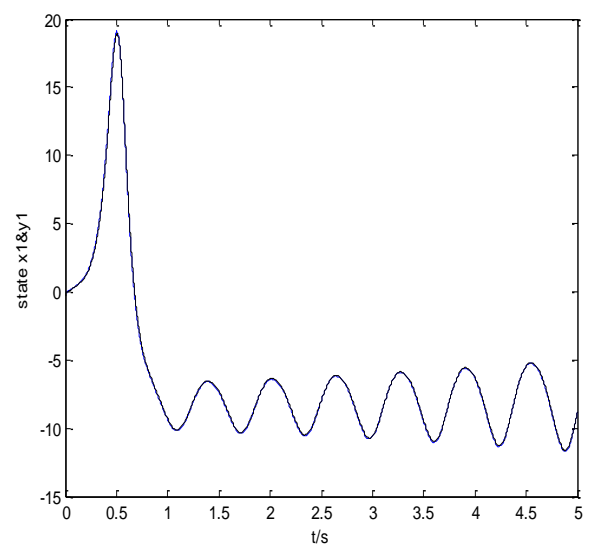

Figure 5. The Curve of State $x 1$ and $\mathbf{y} 1$

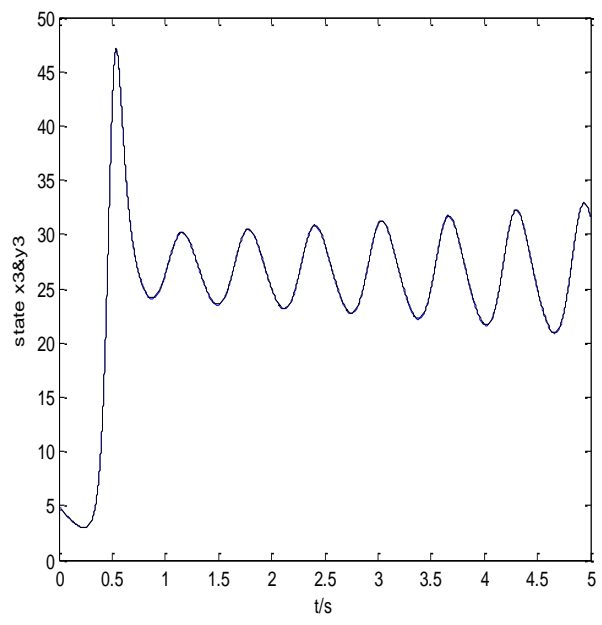

Figure 7. The Curve of State $\times 3$ and y3

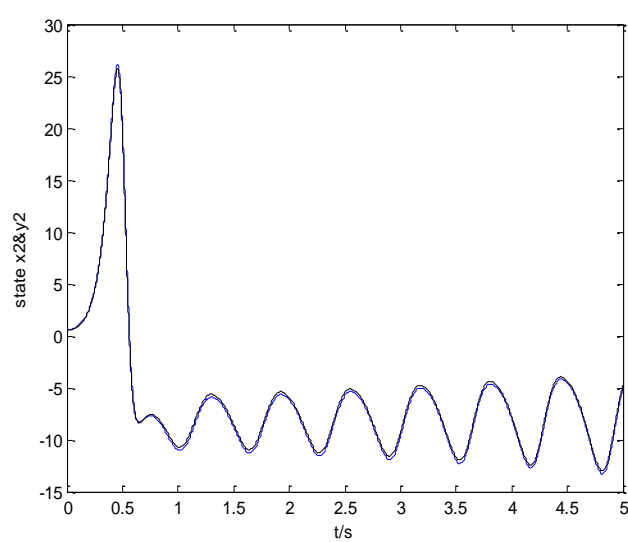

Figure 6. The Curve of State $x 2$ and y2

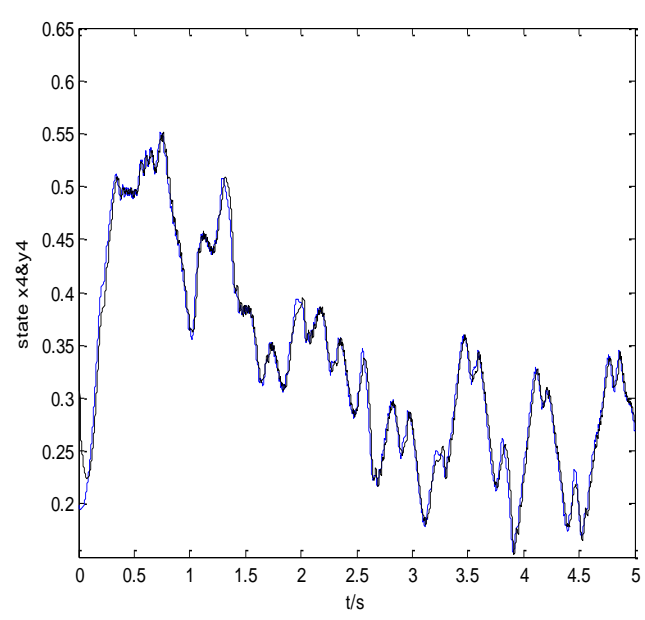

Figure 8. The Curve of State $x 4$ and $y 4$ 
And according to above simulation figures, we can make a conclusion that the response chaotic system can trace the driven chaotic system very well. So the synchronization effect is very good. And the synchronization errors can see following figures.

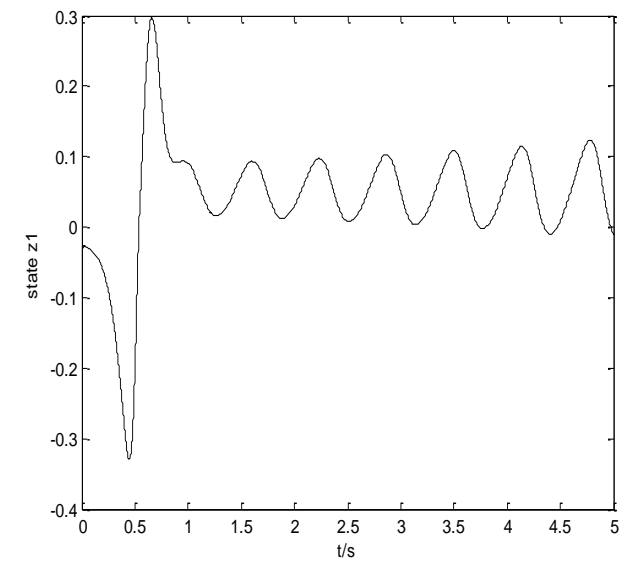

Figure 9. The Curve of State $z 1$

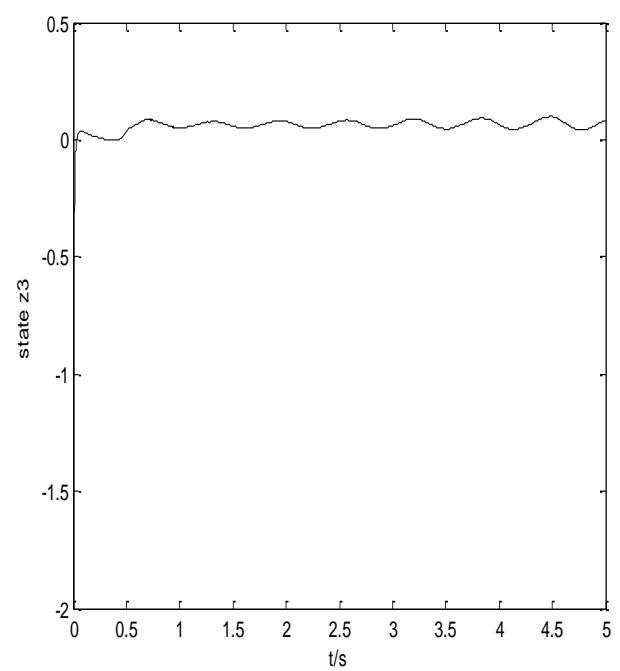

Figure 11. The Curve of State z3

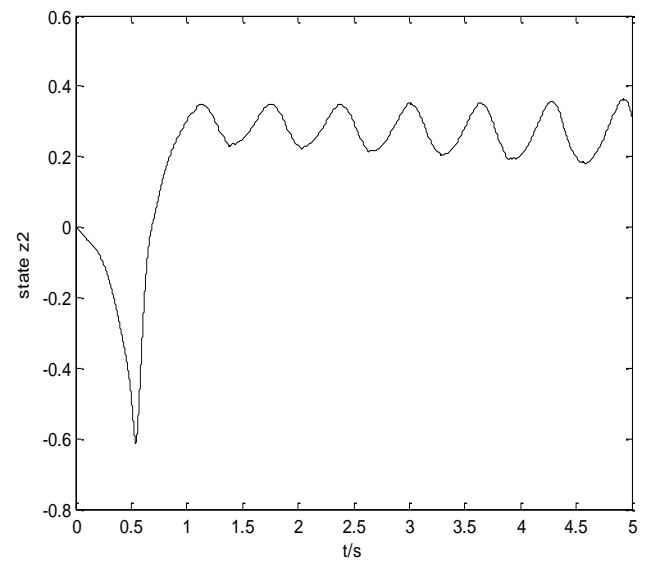

Figure 10. The Curve of State z2

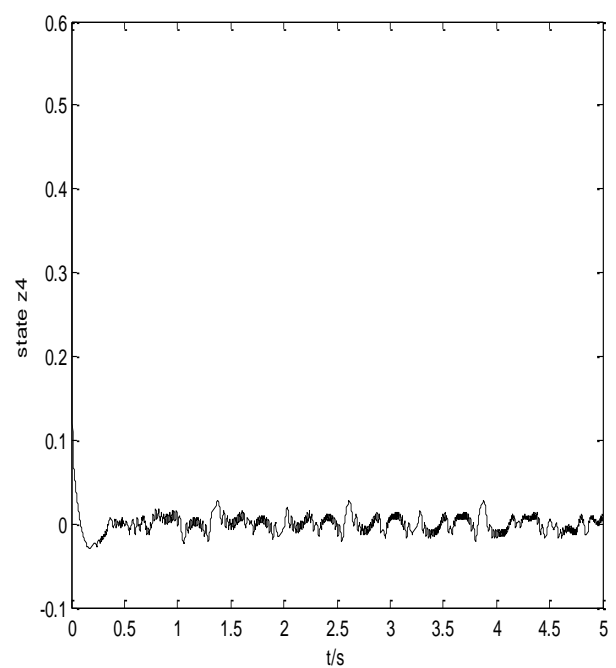

Figure 12. The Curve of State z4

So we can make a conclusion that the synchronization error is converged to a small interval near zero. And if we increase the gain, then the errors can be further reduced.

\section{Numerical Simulation for Synchronization with Sigmoid Function Method}

And we tried the sigmoid function method to do the simulation, and the setting of initial value of systems and adaptive law weights are the same as above soft function method. And we choose $\tau_{i}=0.2$, then simulation results can see following figures. 


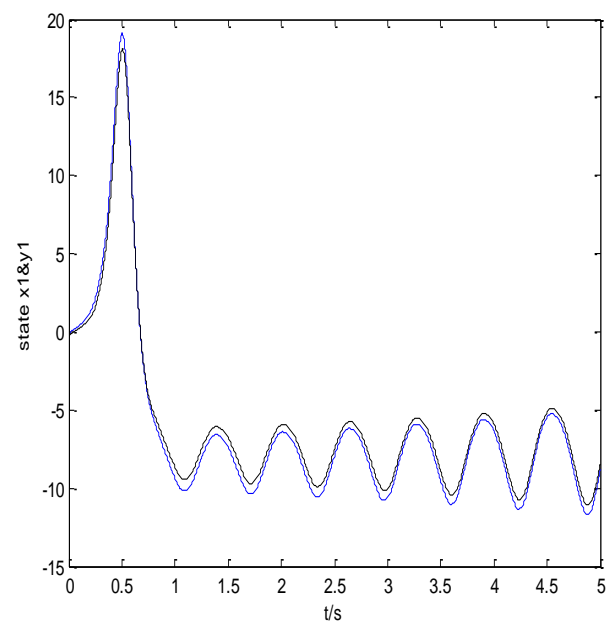

Figure 13. The Curve of State $x 1$ and $\mathbf{y} 1$

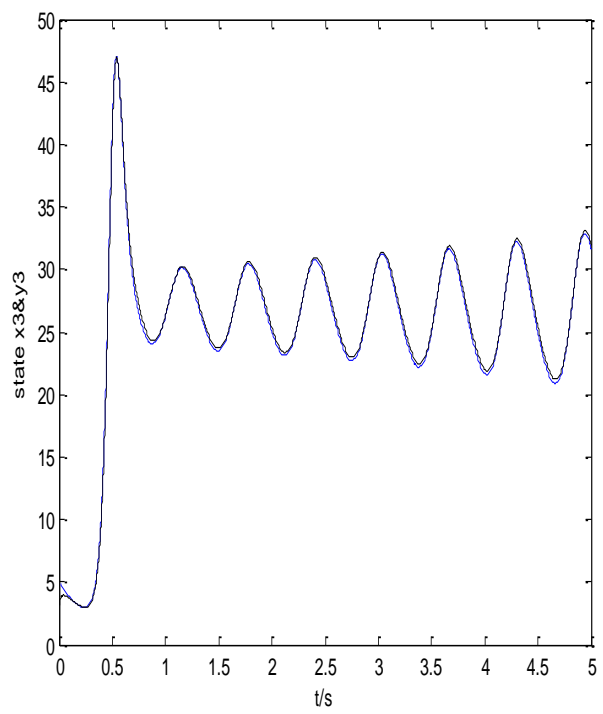

Figure 15. The Curve of State $\times 3$ and y3

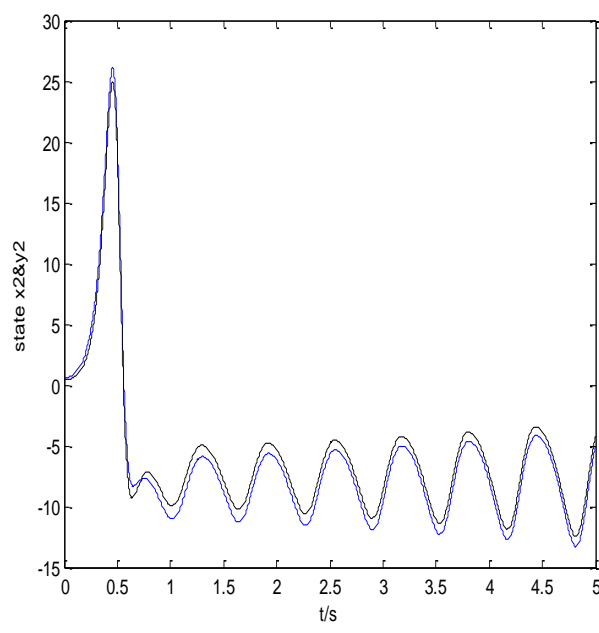

Figure 14. The Curve of State $x 2$ and y2

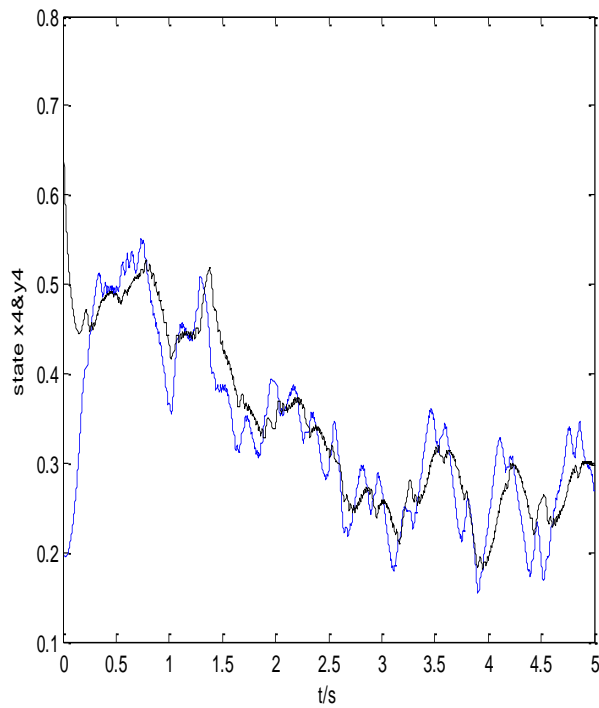

Figure 16. The Curve of State $x 4$ and y4

And according to above simulation result and figures, it is obvious that the synchronization effect is not as good as soft function method with the same setting of controller gains. And we increase $\tau_{i}=2$, then simulation result can see following figures. 


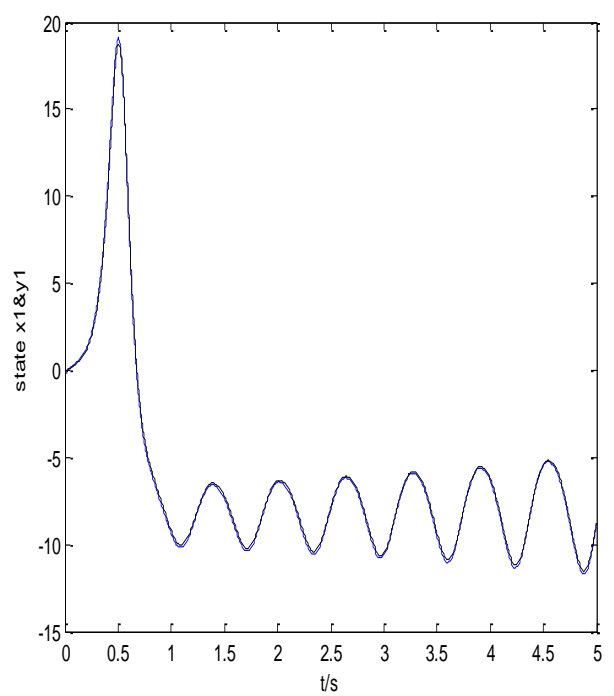

Figure 13. The Curve of State $x 1$ and $y 1$

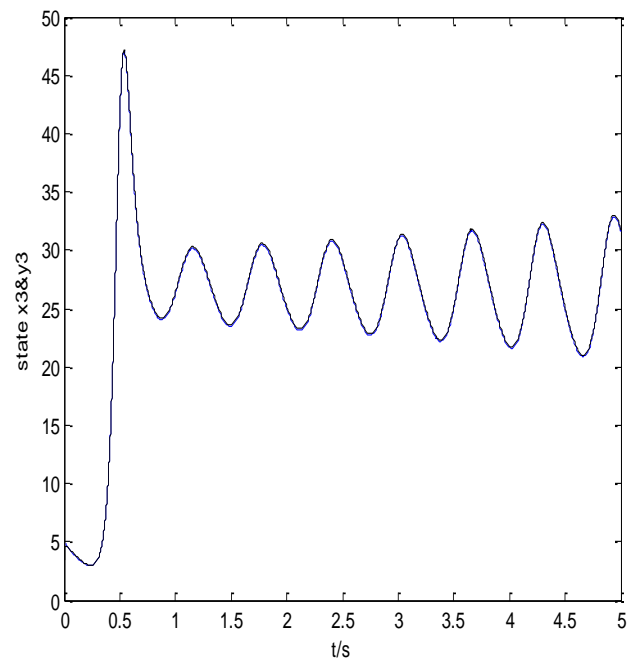

Figure 15. The Curve of State $\times 3$ and y3

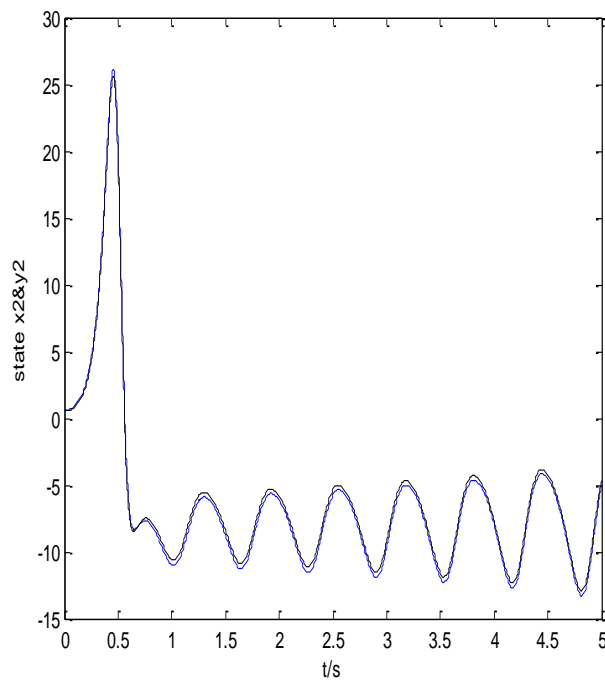

Figure 14. The Curve of State x2 and y2

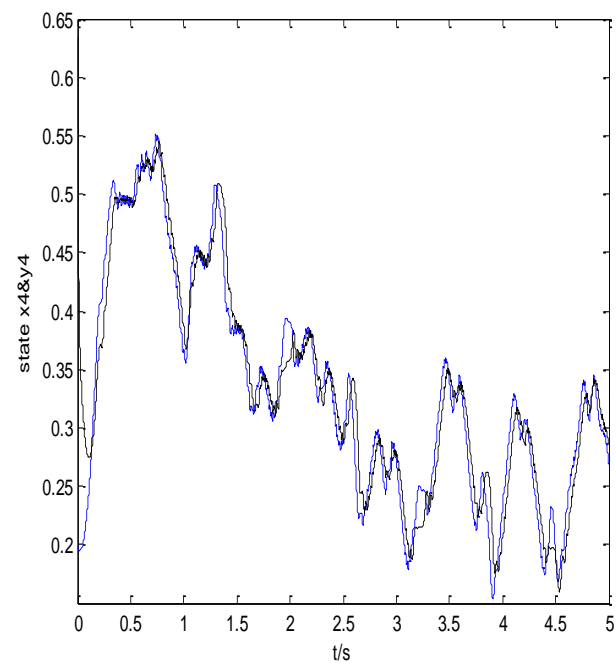

Figure 16. The Curve of State $x 4$ and y4

Then the synchronization effect was improved greatly. So the sigmoid function method was also very useful in some situation of synchronization of chaotic systems.

\section{Conclusion}

In order to realize synchronization of two chaotic systems with uncertain parameters and nonlinearities, two kinds of adaptive and robust method based on soft function and sigmoid function were proposed. The biggest advantage of the proposed two methods is that it do not need the detailed information of the uncertain part of systems and a uniformed adaptive law was designed for every subsystem by using the bounded characteristics of chaotic system. Also by using sigmoid function and soft function method, the chattering problem was greatly suppressed compared with traditional sign 
function method in sliding mode control design. And at last, detailed simulation result shows the rightness and effectiveness of the proposed method.

\section{Acknowledgment}

This paper is supported by Youth Foundation of Naval Aeronautical and Astronautical University of China, National Nature Science Foundation of Shandong Province of China ZR2012FQ010, National Nature Science Foundations of China 61174031, 61004002, 61102167, Aviation Science Foundation of China 20110184 and China Postdoctoral Foundation 20110490266.

\section{References}

[1] I. Kanellakopoulos, P. V. Kokotovic and A. S. Morse, "Systematic design of adaptive controllers for feedback linearizable systems", IEEE transactions on Automatic Control, vol. 36, no. 11, (1991), pp. 1241-1253.

[2] M. Krstic, I. Kanellakopoulos and P. V. Kokotovic, "Nonlinear and Adaptive Control Design", New York: John Wiley and Sons, Inc, vol. 13, no. 5, (1995), pp. 241-253.

[3] M. Kristic, J. Sun and P. V. Kokotovic, "Robust control of nonlinear systems with input unmodeled dynamics", IEEE transactions on Automatic Control, vol. 41, no. 6, (1996), pp. 913-920.

[4] H. N. Agiza and M. T. Yassen, "Synchronization of Rossler and Chen chaotic dynamical systems using active control", Physics Letters A., vol. 278, (2001), pp. 191-197.

[5] M.-c. Ho and Y.-C. Hung, "Synchronization of two different systems by Using generalized active control", Physics Letters A., vol. 301, (2002), pp. 424-428.

[6] E. M. Elabbasy, H. N. Agiza and M. M. El-dessoky, "Adaptive synchronization of System with uncertain parameters", Chaos solitons and fractals, vol. 21, (2004), pp. 657-667.

[7] M. T. Yassen, "Adaptive synchronization of Rosser and lv systems with fully uncertain patameters", Chaos, solitons and fractals, vol. 23, (2005), pp. 1527-1536.

[8] J. H. Park, "Adaptive sysnchronization of Rossler system with uncertain Parameters", Chaos, solitons and fractals, vol. 22, (2005), pp. 1-6.

[9] T.-1. Liao, "Adaptive synchronization of two Lorenz system", Chaos solitons and fractals, vol. 9, no. 9, (1998), pp. 1555-1561.

[10] E. N. Sanchez, J. P. Perez, M. Martinez and G. Chen, "Chaos stabilization: an inverse optimal control approach”, Latin Amer Appl Res: International Journal, vol. 32, (2002), pp. 111-114.

[11] M. Feki, "An adaptive feedback control of linearizable chaotic systems", Chaos, Solitons \& Fractals, vol. 15, (2003), pp. 883-890.

[12] Y. Wang, Z. Guan and X. Wen, "Adaptive synchronization for Chen chaotic system with fully unknown parameters", Chaos, Solitons \&Fractals, vol. 19, (2004), pp. 899-903.

[13] X. Wu and J. Lu, "Parameter identification and backstepping control of uncertain Lv system", Chaos, Solitons \& Fractals, vol. 18, (2003), pp. 721-729.

[14] Y. Yu and S. Zhang, "Adaptive backstepping control of the uncertain Lvu system", Chin Phys., vol. 11, (2002), pp. 1249-305.

[15] C. Wang and S. Ge, "Adaptive backstepping control of uncertain Lorenz system”, International Journal of Bifurc Chaos, vol. 11, (2001), pp. 1115-1119.

[16] E. M. Elabbasy, H. N. Agiza and M. M. El-Dessoky, "Adaptive synchronization of a hyperchaotic system with uncertain parameter", Chaos, Solitons and Fractals, vol. 30, (2006), pp. 1133-1142.

[17] T. Gao, Z. Chen, Z. Yuan and D. Yu, "Adaptive synchronization of a new hyperchaotic system with uncertain parameters", Chaos, Solitons and Fractals, vol. 33, (2007), pp. 922-928.

[18] X. Wang, J. Lei and C. Pan, "Trigonometric RBF neural robust controller design for a class of nonlinear system with linear input unmodeled dynamics", Applied Mathematics and Computation, 185(2007)pp.989-1002.

[19] Seung-Hwan Kim, Yoon-Sik Kim, Chanho Song, A robust adaptive nonlinear control approach to missile autopilot design, Control engineering practice, 12(2004) pp. 149-154.

[20] C.C. Hwang, J.Y. Hsieh, R.S. Lin. A linear continuous feedback control of Chua's circuit, Chaos, Solitons, Fractals 8 (1997) 1507-1515.

[21] G.H. Li. Generalized projective synchronization between Lorenz system and Chen's system, Chaos, Solitons, Fractals 32 (2007) 1454-1458. 


\section{Authors}

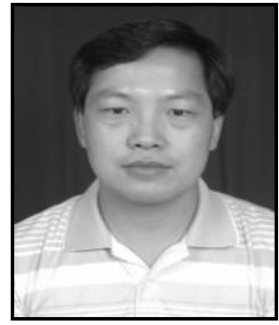

Zhanlei Shang(1974-) was born in Yuzhou city of Henan province of China. He received his bachelor's degree in 1996 from Henan Normal University and his major is physical education and received his major's degree in 2000 from Huazhong University of Science and Technology and his major is computer technology. He is an Associate Professor and a Director of Computer Center of Zhengzhou University of Light Industry. His current research interests is database and network. He published more than 20 academic papers, and 5 of them were retrieved by EI. About 10 computer textbooks were issued under his general editorship, 3 of them were respectively awarded the Eleventh Five-Year Plan and the Twelfth Five-Year Plan for National Teaching Materials. In addition, He had taken charge of and participated in 8 scientific projects and 6 education reform projects.

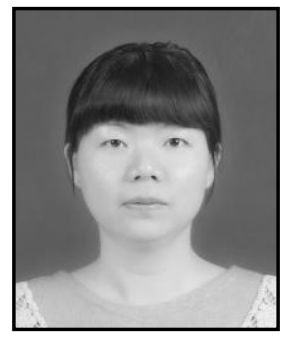

Jing Cheng, She was born in 1977 in Luoyang city of Henan province of China. She received her BS in Computer Application from Zhengzhou University of Light Industry, Zhengzhou, China, in 2000.She got her MS in Computer Technology from Huazhong University of Science and Technology, Wuhan, China, in 2005. Now she is an Associate Professor in the Engineer Training Center at Zhengzhou University of Light Industry. Her research interests include computer application technology and computer network.

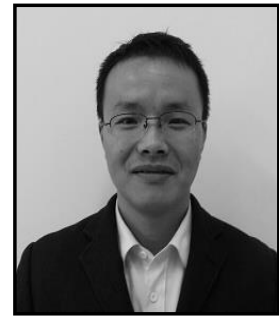

Junwei Lei, He was born in 1981 in Chibi of Hubei province of China and received his Doctor degree in Guidance, Navigation and Control in 2010 from Naval Aeronautical and Astronautical University, Yantai of China. Her present interests are control theory, chaotic system control, aircraft control and adaptive control.

He was promoted to be a lecture of NAAU in 2010. His typical book named Nussbaum gain control technology of supersonic missiles was published in 2013 in China.

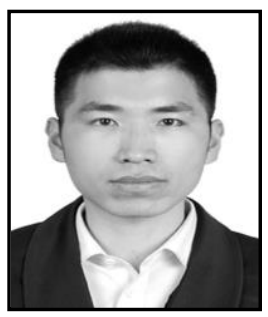

Ruiqi Wang, He was born in 1980 in Xinxiang City, Henan province of China. He received his Doctor degree in Guidance, Navigation and Control in 2011 from Naval Aeronautical and Astronautical University, Yantai of China. He graduated from Naval Aeronautical and Astronautical University, Yantai of China in 2001 and received his bachelor's degree with the major of missile control and test. After that he continued his study in this school and received his master's degree and doctor's degree in 2006 and 2011 respectively. He has published more than 20 papers, where 7 papers was indexed by Ei. His present interests are control theory, missile control and bilateral control. 
International Journal of Signal Processing, Image Processing and Pattern Recognition Vol. 9, No. 6 (2016) 